\title{
Imaging Features of Artificial Intelligence Algorithm in the Analysis of Cerebral Protective Effect of Craniotomy Hematoma Removal under Propofol Anesthesia in Patients with Chronic Subdural Hematoma
}

\author{
Manyun Bai ${ }^{D},{ }^{1}$ Yufang Li $(\mathbb{D})^{1}$ Qian Zhao $\mathbb{D}^{1},{ }^{1}$ and Renzhong Guo $\left.{ }^{2}\right)^{2}$ \\ ${ }^{1}$ Department of Anesthesiology, The Fourth Hospital of Changsha (Changsha Hospital of Hunan Normal University), \\ Changsha 410006, Hunan, China \\ ${ }^{2}$ Department of Otorhinolaryngology-Head and Neck Surgery, \\ Changsha Central Hospital (Changsha Central Hospital Affiliated to University of South China), Changsha 410004, \\ Hunan, China \\ Correspondence should be addressed to Renzhong Guo; zxh@cpu.edu.cn
}

Received 10 July 2021; Accepted 16 August 2021; Published 6 September 2021

Academic Editor: Gustavo Ramirez

Copyright (c) 2021 Manyun Bai et al. This is an open access article distributed under the Creative Commons Attribution License, which permits unrestricted use, distribution, and reproduction in any medium, provided the original work is properly cited.

\begin{abstract}
Objective. The aim of this work was to study the cerebral protective effect of craniotomy hematoma removal under propofol anesthesia based on the artificial intelligence algorithm analysis of the changes in imaging characteristics of chronic subdural hematoma (CSDH) patients. Methods. A total of $60 \mathrm{CSDH}$ patients who were treated in hospital were recruited and were randomly rolled into an experimental group and a control group, with 30 people in each group. Patients in the experimental group were treated with propofol anesthesia + craniotomy hematoma removal, while those in the control group were treated with conventional anesthesia + craniotomy hematoma removal. Head CT examinations were performed on the next day, one week, one month, three months, and six months after the operation. A two-dimensional empirical mode decomposition (BEMD) algorithm was used for edge detection and denoising of brain CT images of CSDH patients. Then, the amount of hematoma was calculated, and the Markwalder grading was performed to evaluate the neurological function. The number of recurrence and reoperation cases within six months of follow-up was collected. Results. 1. The quality of CT images was remarkably improved after processing with artificial intelligence algorithms. 2. The amount of hematoma in the experimental group was remarkably lower than that in the control group at January, March, and June after surgery (12.89 \pm 2.12 VS $20.32 \pm 16.41 ; 7.55 \pm 4.13$ VS $15.88 \pm 14.22 ; 3.39 \pm 3.79$ VS $6.55 \pm 3.69, P<0.05)$. 3. The experimental group was remarkably better than the control group in Markwalder grading three months and six months after the operation $(P<0.05)$. Conclusion. Artificial intelligence algorithm had good effect on the brain CT image processing of $\mathrm{CSDH}$ patients, and craniotomy hematoma removal under propofol anesthesia had an ideal brain protection effect.
\end{abstract}

\section{Introduction}

Chronic subdural hematoma (CSDH) is a common neurosurgical disease. Most of the patients are elderly people. In recent years, with the increasing trend of population aging, the incidence of CSDH has been rising. In the early stage of $\mathrm{CSDH}$, due to the small hematoma and relatively hidden symptoms, it is easily despised by patients and their families, leading to delayed treatment. With the increasing amount of hematoma, the clinical manifestations include vomiting, dizziness, and other intracranial hypertension symptoms, with prominent headache. Some patients are with progressive dementia, memory loss, mental retardation, and other neurological impairment symptoms. A few may have typical symptoms such as paralysis of limbs, speech disorders, and epilepsy, and even coma in severe cases [1]. 
The etiology of CSDH is abnormal subdural accumulation of degradation products of liquefied blood. If timely diagnosis and treatment are carried out, the prognosis of this disease is relatively good [2]. Surgical treatment is currently the main treatment for CSDH. Craniotomy hematoma removal and hematoma drainage are the two most common surgical methods for CSDH at present [3]. Craniotomy hematoma removal, as a traditional and classic surgical method, is used to remove hematoma under direct vision and to deal with various emergencies during operation. Therefore, it is widely used in clinical practice [4]. Hematoma suction and drainage has the advantages of being minimally invasive, involves simple operation, does not need general anesthesia, and is also a common surgical method. With the continuous maturity of surgical techniques, surgical treatment has achieved good therapeutic effects $[5,6]$.

It is inevitable to anesthetize patients during surgical treatment. Propofol is currently widely used for induction, maintenance of anesthesia, and ICU sedation [7]. Propofol is often used in CSDH patients as the anesthesia inducer during surgical treatment. However, there is no definite conclusion about the effect of propofol on the brain. Studies suggested that propofol can cause forgetting and even lead to impaired memory function [8]. However, there is also a view that propofol has no effect on the learning ability and memory function of experimental animals. The reason why these conclusions are so inconsistent may be that the anesthesia effect is influenced by many factors such as oxygen concentration, age, and research methods [9]. Some studies indicated that hypoxic exposure can affect the proteins associated with learning and memory, thus further affecting the brain's ability to learn and remember. Many clinical studies revealed that anesthesia, hypotension, pathological shunt, thoracic surgery, single lung ventilation, and other conditions may cause hypoxia [10]. In a word, the effects of propofol on the brain still need to be further explored and studied.

At present, brain CT is the most effective, accurate, and major method for diagnosis of CSDH patients. Traditional $\mathrm{CSDH}$ diagnosis methods manually judge the disease condition and process based on doctors' CT images. At present, with the extensive application of brain CT and the increasing number of $\mathrm{CSDH}$ patients, doctors are under increasing pressure to read the images. This traditional method of diagnosis is often affected by the subjective judgment of doctors and the level of medical experience, so it often leads to misdiagnosis and missed diagnosis, which is extremely detrimental to the prognosis and treatment of the disease [11]. Therefore, it is very important and urgent to develop a rapid and accurate auxiliary diagnostic system. With the continuous development of computer technology, some image processing technology is becoming more and more perfect and mature. These techniques have also found some applications in medical image processing. Some scholars applied the computer diagnostic system for the diagnosis of glioma, Alzheimer's disease, lung cancer, and other diseases [12]. Artificial intelligence algorithm is one of the most important parts in computing and auxiliary diagnosis system. The typical structure of the computer aided diagnosis system includes image preprocessing, feature extraction and selection, as well as classification. The image preprocessing process may include image standardization and noise removal. The artificial intelligence algorithm is designed for image preprocessing, feature extraction and selection, and classification modules [13]. At present, there are few studies on the application of artificial intelligence algorithm in $\mathrm{CSDH}$ disease diagnosis. In this research, the artificial intelligence algorithm was used to process the CT images of CSDH patients. The effect of craniotomy hematoma removal under propofol anesthesia on the brain function of patients was explored. It was expected to provide reference and basis for the treatment of related diseases and the application of artificial intelligence algorithm in disease diagnosis of $\mathrm{CSDH}$ patients.

In this research, a newly emerging image processing algorithm, Bidimensional Empirical Mode Decomposition (BEMD), was used to perform a series of processing such as feature extraction and denoising of BRAIN CT images of CSDH patients. On this basis, the recovery of brain function of CSDH patients after the removal of craniotomy hematoma under propofol anesthesia was explored with the amount of postoperative hematoma, Markwalder grade, second operation rate and recurrence rate as the starting point, so as to provide reference and basis for clinical application of BEMD algorithm and removal of craniotomy hematoma under propofol anesthesia in the treatment of $\mathrm{CSDH}$.

\section{Materials and Methods}

2.1. Research Objects. A total of $60 \mathrm{CSDH}$ patients who have been treated in hospital from January 2018 to February 2020 were recruited. There were 44 male patients and 16 female patients, with an average age of $70.77 \pm 12.33$ years old. All patients were diagnosed as CSDH by CT and underwent craniotomy hematoma removal. Exclusion criteria were defined as patients with preoperative cerebral hernia or abnormal coagulation function and those who did not complete the follow-up. All the patients were randomly rolled into an experimental group and a control group, with 30 people in each group. Patients in the experimental group were treated with propofol anesthesia + craniotomy hematoma removal, while those in the control group were treated with conventional anesthesia + craniotomy hematoma removal. All the studies in this research obtained informed consent and met the standards and requirements of medical ethics.

2.2. Anesthesia Methods. Experimental group (group A): induction of propofol $1-2 \mathrm{mg} / \mathrm{kg}$, sufentanil $0.2-0.4 \mu \mathrm{g} / \mathrm{kg}$, and cis atracurium $0.1-0.2 \mathrm{mg} / \mathrm{kg}$. After the patient was unconscious and the muscles were relaxed, the laryngeal mask was placed and mechanical ventilation was performed. Intravenous pump propofol-remifentanil was used for the maintenance of anesthesia (propofol $4-6 \mathrm{mg} / \mathrm{kg} \cdot \mathrm{h}$ and remifentanil 5-10ug/kg.h). Propofol was used to deepen or lighten the anesthesia during the operation. Intermittent 
intravenous injection of cis atracurium 2-4 mg/time was done to meet the needs of the surgeon.

Control group (group B): sevoflurane inhalation induction was performed with the tidal volume method. The fresh air valve was closed, the APL valve was opened, and the breathing bag was empty. The sevoflurane was turned to $8 \%$ and the oxygen to $6 \mathrm{~L} / \mathrm{min}$. When the respirator was full, the patient inhaled deeply and exhaled. At the end of exhalation, mask was placed over the patient's face, who would breathe autonomously. When the patient was unconscious, the sevoflurane concentration was adjusted to 4-5\%. Intravenous injections of sufentanil $0.2-0.4 \mu \mathrm{g} / \mathrm{kg}$ and cis atracurium $0.1-0.2 \mathrm{mg} / \mathrm{kg}$ were performed. After $3-5 \mathrm{~min}$ of manual breathing, the laryngeal mask was inserted, and mechanical ventilation was performed. Group B was maintained with sevoflurane-remifentanil, sevoflurane $1-2 \%$, and remifentanil 5-10 $\mu \mathrm{g} / \mathrm{kg} \cdot \mathrm{h}$. The anesthetic depth was controlled by adjusting the sevoflurane concentration during the operation. An intermittent intravenous injection of cis atracurium $2-4 \mathrm{mg} /$ time was given to meet the surgical needs.

2.3. Treatment Methods. The surgical approach was determined based on the site of bleeding, and the frontotemporal inquisit-shaped incision or the horseshoe incision of the corresponding site of bleeding was usually used for supratentorial hematoma. Under the microscope, the lateral fissure was separated, and the basal ganglia hematoma was removed through the insular approach. Or, the cortical fistula entered the hematoma cavity where the hematoma was closest to the cortex. It was necessary to avoid important functional areas during stoma. The hematoma was cleared under the microscope, and after strict hemostasis, the head was closed after hemostasis materials were put into the operative cavity. The most common approach for hemorrhage in the infratentorial cerebellar hemisphere was the suboccipital posterior median or paracentral median approach. During craniotomy, the foramen magnum was bitten open for decompression. The hematoma was removed by the same method as above, hemostasis, and routine craniotomy. In terms of indwelling and drainage, since the surgeon performing the operation was different, the operation was also different, so did the intraoperative indwelling and drainage. If small amounts of hematoma remains were difficult to be removed during the operation, the hematoma cavity can be placed for drainage. Usually, only epidural or subcutaneous drainage was required, or no drainage was required. For postoperative management, the CT scan of the head should be performed as soon as possible (within six hours) after surgery if the condition permitted to determine the residual hematoma and whether there was rebleeding. Depending on the situation, head CT reexamination was made. The drainage tube was usually pulled out after about three days, and if there was ventricular drainage at the same time, the ventricular drainage tube should not be kept for more than one week.
2.4. Brain CT Image Processing. Denoising processing: it is assumed that an image $f(x, y)$ is an array of $M \times N$, the processed image is $g(x, y)$, and its gray level is determined by the average value of the gray levels of several pixels in the area $(x, y)$. The processed image is represented as follows:

$$
g(x, y)=\frac{1}{M} \sum_{(i, j) \in s} f(i, j) .
$$

Among them, $x, y=0,1,2 \ldots, N-1 . S$ is the field collection centered on point $(x, y)$, and $M$ is the total number of internal coordinate points in $S$.

For multiple images, the original image is $f(x, y)$ and the image noise is $n(x, y)$, then the noisy image $g(x, y)$ is expressed as follows:

$$
g(x, y)=f(x, y)+n(x, y)
$$

If the noises are not correlated with each other and the mean value is zero, then

$$
f(x, y)=E[g(x, y)] .
$$

$E[g(x, y)]$ is the expected value of $g(x, y)$, and following are the equations after averaging of $M$ noisy images:

$$
\begin{aligned}
f(x, y) & =E[g(x, y)] \sim \bar{g}(x, y) \\
& =\frac{1}{M} \sum_{i=1}^{M} g_{i}(x, y), \\
\delta^{2}{ }_{\bar{g}(x, y)} & =\frac{1}{M} \delta_{n(x, y) .}^{2}
\end{aligned}
$$

$\delta_{\bar{g}(x, y)}^{2}$ and $\delta_{n(x, y)}^{2}$ are the variance of $\bar{g}$ and $n$ at the point $(x, y)$.

Edge detection is as follows:

$$
\begin{aligned}
& \psi^{1}(x)=\frac{\mathrm{d} \theta(x)}{\mathrm{d} x}, \\
& \psi^{2}(x)=\frac{\mathrm{d}^{2} \theta(x)}{\mathrm{d} x^{2}} .
\end{aligned}
$$

Equation (7) is defined as follows:

$$
\begin{aligned}
w^{1} f(s, x) & =f^{*} \psi_{s}^{1}(x), \\
w^{2} f(s, x) & =f^{*} \psi_{s}^{2}(x) . \\
w^{1} f(s, x) & =f^{*}\left(s \frac{\mathrm{d} \theta_{s}}{\mathrm{~d} x}\right)(x) \\
& =s \frac{\mathrm{d}}{\mathrm{d} x}\left(f^{*} \theta_{s}\right)(x), \\
w^{1} f(s, x) & =f^{*}\left(s^{2} \frac{\mathrm{d}^{2} \theta_{s}}{\mathrm{~d} x^{2}}\right)(x) \\
& =s^{2} \frac{\mathrm{d}^{2}}{\mathrm{~d} x^{2}}\left(f^{*} \theta_{s}\right)(x) .
\end{aligned}
$$


The film value of the discrete dyadic wavelet transforms $W_{2^{j}}^{1, d} f(n, m), W_{2^{j}}^{2, d} f(n, m)$ at point $(n, m)$ is as follows:

$$
M_{2^{j}}^{d} f(n, m)=\sqrt{\left.W_{2^{j}}^{1, d} f(n, m)\right|^{2}+\left|W_{2^{j}}^{2, d} f(n, m)\right|^{2}} .
$$

The phase angle is as follows:

$$
A_{2^{j}}^{d} f(n, m)=\arg \tan \left(\frac{W_{2^{j}}^{2, d} f(n, m)}{W_{2^{j}}^{1, d} f(n, m)}\right) .
$$

2.5. Observation Indexes. The preoperative information of patients (gender, age, subdural hematoma volume, midline deviation degree, Markwalder grade, etc.) was collected and compared. After the operation, the patients were followed up for six months. CT examination was performed on the next day, one week, one month, three months, and six months after the operation, and hematoma volume was calculated again based on CT examination. Markwalder grading evaluation was carried out, and the specific content of grading is shown in Table 1 . The number of cases with recurrence and reoperation was counted.

2.6. Statistical Methods. All data analysis was completed by SPSS 19.0. Measurement data were expressed by $(\bar{x} \pm s)$, an independent sample $t$-test was used, and comparison between groups was completed by the Chi-square test. Nonparametric rank of independent samples and Mann-Whitney $U$ test were used for descriptive samples, and $P<0.05$ was considered statistically substantial.

\section{Results}

3.1. Comparison of General Information. The general preoperative data of the two groups of patients are shown in Tables 2, 3, and Figure 1. There was no great difference between the two groups of patients in general information such as age, hematoma side, hematoma density, hematoma volume, hematoma thickness, midline deviation, and Markwalder grading, and the difference between the groups was not statistically substantial $(P>0.05)$, suggesting that the two groups of patients were comparable.

3.2. Brain CT Results. The experimental group was taken as an example to display the original brain CT images before surgery, the day after surgery and one week, one month, three months, and six months after surgery, and the images were processed by the artificial intelligence algorithm, as illustrated in Figure 2. The quality of the image processed by the artificial intelligence algorithm was remarkably higher than that of the unprocessed image. In addition, the images processed by the artificial intelligence algorithm can better, clearer, and more accurately reflect each stage of the two groups after surgery.

3.3. Postoperative Hematoma Volume. The hematoma volume of the two groups of patients the next day, one week, one month, three months, and six months after surgery and the comparison results between the two groups are shown in Figure 3. There was no considerable difference in the amount of hematoma between the two groups on the next day and one week after surgery (42.81 \pm 7.12 VS $43.31 \pm 7.56$ and $21.44 \pm 2.24$ VS $22.33 \pm 1.56, P>0.05)$. The hematoma volume of the experimental group was remarkably lower than that of the control group one month, three months, and six months after surgery (12.89 \pm 2.12 VS $20.32 \pm 16.41$; $7.55 \pm 4.13$ VS $15.88 \pm 14.22 ; 3.39 \pm 3.79$ VS $6.55 \pm 3.69$, $P<0.05)$.

3.4. Postoperative Markwalder Grading. Postoperative Markwalder grades of patients in both groups are shown in Figure 4 and Table 4 . There was no considerable difference in Markwalder grading between the two groups the next day, one week, and one month after surgery, and there was no statistical significance between the two groups $(P>0.05)$. Markwalder grading in the experimental group was remarkably lower than that in the control group at three and six months after surgery, and the difference between the two groups was statistically substantial $(P<0.05) .3$ months and 6 months after operation, the Markwalder grading results of the experimental group were as follows: 3 months: 23 cases of grade 0,8 cases of grade 1,1 case of grade 2, 0 cases of grade 3 , and 0 cases of grade $4 ; 6$ months: 30 cases of grade 0 , 2 cases of grade 1, 0 cases of grade 2, 0 cases of grade 3, and 0 cases of grade 4 . The results of Markwalder grading in the experimental group 3 months and 6 months after operation in the control group were as follows: 3 months: 13 cases of grade 0,18 cases of grade 1,1 case of grade 2, 0 cases of grade 3 , and 0 cases of grade 4; 6 months: 22 cases of grade 0,9 cases of grade 1, 1 case of grade 2, 0 cases of grade 3 , and 0 cases of grade 4 . From this, the number of patients with Markwalder grade 0, 3, and 6 months after operation in the experimental group was significantly higher than that in the control group. Comparison between the two groups showed $P=0.017<0.05$ and $P=0.011<0.05$.

3.5. Postoperative Recurrence in the Two Groups. The comparison of postoperative recurrence between the two groups of patients is shown in Figure 5. Only one patient in group A had postoperative hematoma increase, and the recurrence rate was $3.3 \%$. In group B, postoperative hematoma increased in seven patients, and the recurrence rate was $23.3 \%$. The difference between the two groups was obvious and statistically substantial, $P<0.05$.

3.6. Comparison of the Reoperation Rate between the Two Groups. The comparison results of the reoperation rate between the two groups are shown in Figure 6. No patient in group A underwent a second operation, and the reoperation rate was $0 \%$. In group $B$, five patients underwent a second operation, and the reoperation rate was $16.6 \%$. The comparison of the reoperation rate between the two groups was considerable and statistically substantial, $P<0.05$. 
TABLE 1: Markwalder's neural function grading.

\begin{tabular}{lc}
\hline Grade & Symptoms \\
\hline 0 & There were no signs of neurological function. \\
1 & Neurological symptoms were mild, such as dizziness, headache, and mild neurological impairment. \\
2 & Sleepiness, misorientation, and moderate neurological dysfunction, such as mild hemiplegia. \\
3 & Shallow comas and severe neurological disorders, such as hemiplegia. \\
4 & Severe coma, sensitivity to pain, and cerebral and cortical stiffness. \\
\hline
\end{tabular}

TABLE 2: General information of the two groups of patients.

\begin{tabular}{lcc}
\hline Items & Experimental group (group A) & Control group (group B) \\
\hline & $n=30$ & $n=30$ \\
Gender (male) & 27 & 29 \\
Age (years) & $71.8 \pm 11.1$ & $68.8 \pm 14.3$ \\
Hematoma side (right) & 17 & 14 \\
Hematoma density (uniform) & 18 & 12 \\
Hematoma volume (mL) & $117.6 \pm 17.8$ & $116.5 \pm 21.09$ \\
Thickness of hematoma (cm) & $2.35 \pm 0.33$ & $2.546 \pm 0.589$ \\
Midline deviation (cm) & $1.05 \pm 0.32$ & $1.21 \pm 0.31$ \\
\hline
\end{tabular}

TABle 3: Preoperative Markwalder grading and comparison of basic data between the two groups of patients.

\begin{tabular}{lcc}
\hline Items & $P$ & $X_{2} / t / Z$ \\
\hline Gender & 0.184 & 0.668 \\
Age & 0.803 & 0.425 \\
Hematoma side & 0.259 & 0.611 \\
Hematoma density & 1.004 & 0.316 \\
Hematoma volume & 0.151 & 0.88 \\
Thickness of hematoma & -0.413 & 0.681 \\
Midline deviation & -0.886 & 0.379 \\
& -0.777 & 0.437 \\
\hline
\end{tabular}

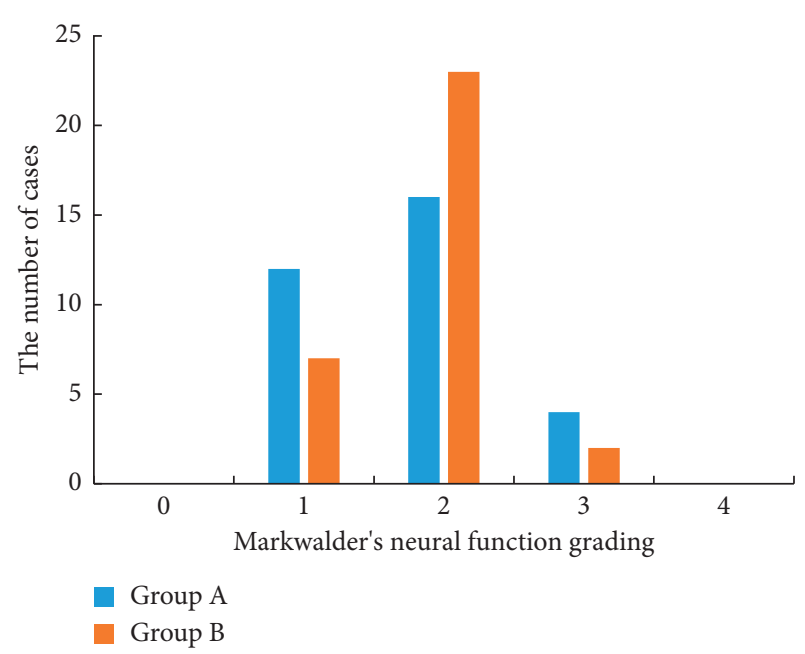

Figure 1: Preoperative Markwalder grading and comparison of basic data between the two groups of patients.

\section{Discussion}

In recent years, the aging of Chinese population has been increasing. The incidence of some diseases with the elderly as the main morbidity group continues to rise, and $\mathrm{CSDH}$ is one of them. As a common chronic neurosurgical disease, the early symptoms of CSDH are relatively hidden and generally not suitable for diagnosis. As a result, the best time for disease treatment is missed, leading to severe sequelae such as hemiplegia, neurological impairment, and aphasia [14]. The number of CSDH patients admitted to our hospital in the recent years has also been increasing year by year, which is consistent with the above-mentioned studies.

At present, brain CT is the most accurate and effective method to diagnose CDSH. Clinically, the development and prognosis of the disease are generally judged based on the brain CT and doctors' own experience and professional knowledge to develop the treatment plan. This method is greatly influenced by the subjective understanding of doctors, which often leads to misdiagnosis and missed diagnosis, and the diagnosis results of different doctors are also different. In addition, the number of patients is increasing, and CT technology is being used more and more widely [15]. With the continuous development of computing technology and artificial intelligence, many scholars focused on the rapid auxiliary diagnosis system based on medical images and achieved certain research results [16]. For example, the computer diagnostic system is applied to the diagnosis of glioma, Alzheimer's disease, lung cancer, and other diseases 


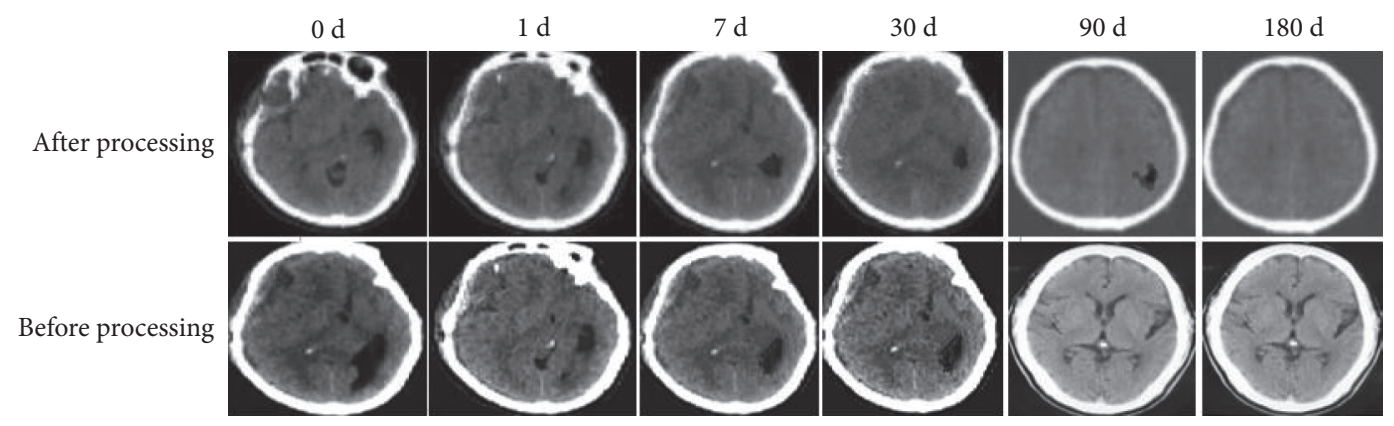

FIgURE 2: Brain CT display of CDSH patients at various stages before and after being processed by the artificial intelligence algorithm.

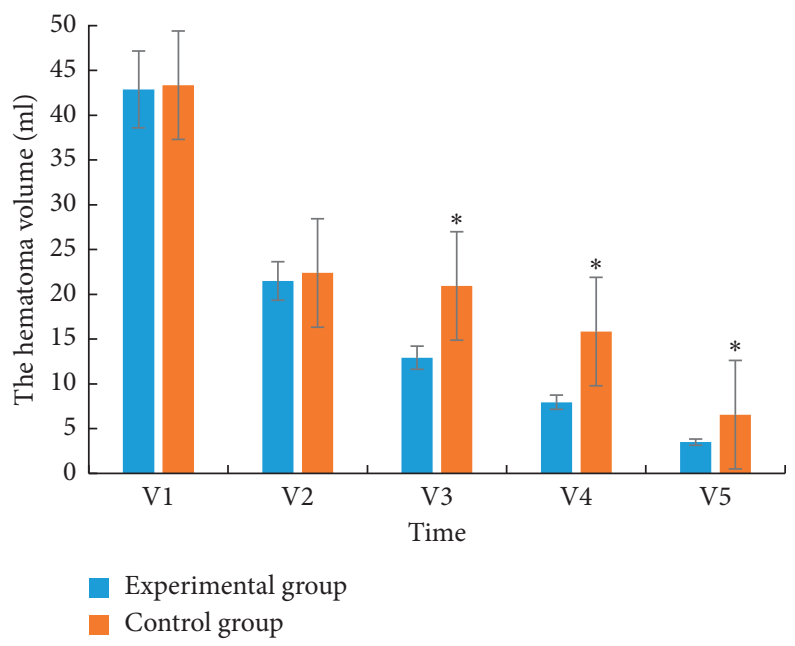

Figure 3: Comparison of the hematoma volume between the two groups of patients in each period. Note. V1, V2, V3, V4, and V5 represented the CSDH hematoma volume of the experimental group and the control group on the next day, one week, one month, three months, and six months after surgery, respectively. ${ }^{*}$ means statistical significance compared with the experimental group, $P<0.05$.

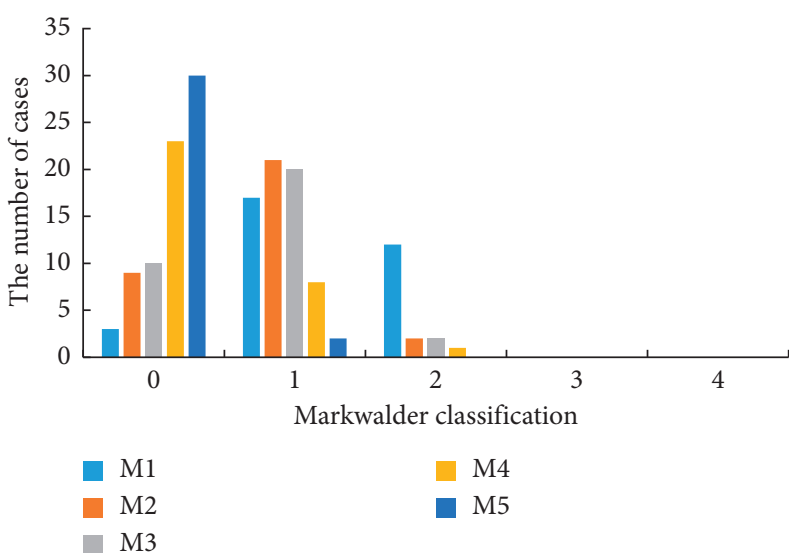

(a)

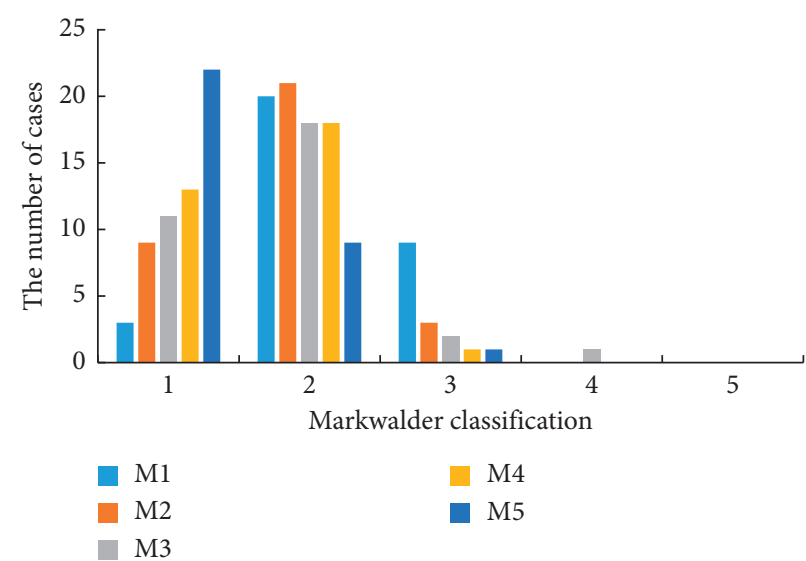

(b)

FiguRE 4: Statistical analysis of the Markwalder score results in each postoperative period in the two groups: (a) experimental group; (b) control group. Note. M1, M2, M3, M4, and M5 represented the Markwalder grading of CSDH patients in the experimental group and the control group on the next day and after one week, one month, three months, and six months after surgery, respectively.

[17-19]. In this research, a newly emerging image processing algorithm Bidimensional Empirical Mode Decomposition (BEMD) was used to perform a series of processing such as feature extraction and denoising of BRAIN CT images of $\mathrm{CSDH}$ patients. The results showed that CT images processed by BEMD were clearer and had higher image quality. 
TABLE 4: Markwalder classification comparison results of the two groups at each time period.

\begin{tabular}{llr}
\hline Time & $\mathrm{Z}$ & \multicolumn{1}{c}{} \\
\hline M1 & -0.655 & 0.506 \\
M2 & -0.183 & 0.855 \\
M3 & -0.109 & 0.913 \\
M5 & -2.395 & 0.017 \\
\hline
\end{tabular}

Note. M1, M2, M3, M4, and $M 5$ represented the Markwalder grading of CSDH patients in the experimental group and the control group on the next day and after one week, one month, three months, and six months.

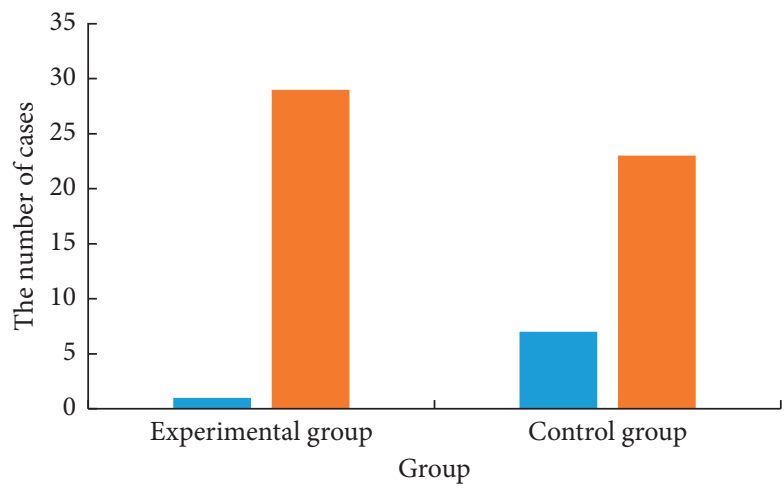

Recurrence

No recurrence

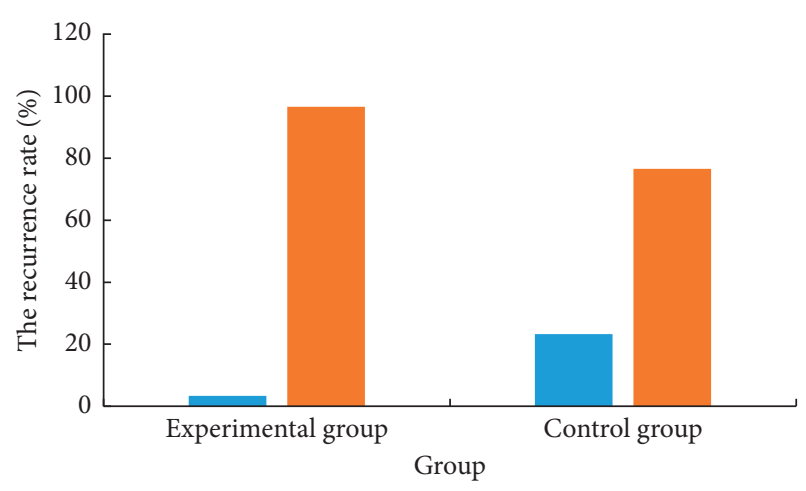

Recurrence

No recurrence

(a)

(b)

FiguRE 5: Comparison of postoperative recurrence between the two groups. (a) recurrence number; (b) recurrence rate. * means the statistical significance compared with the experimental group, $P<0.05$.

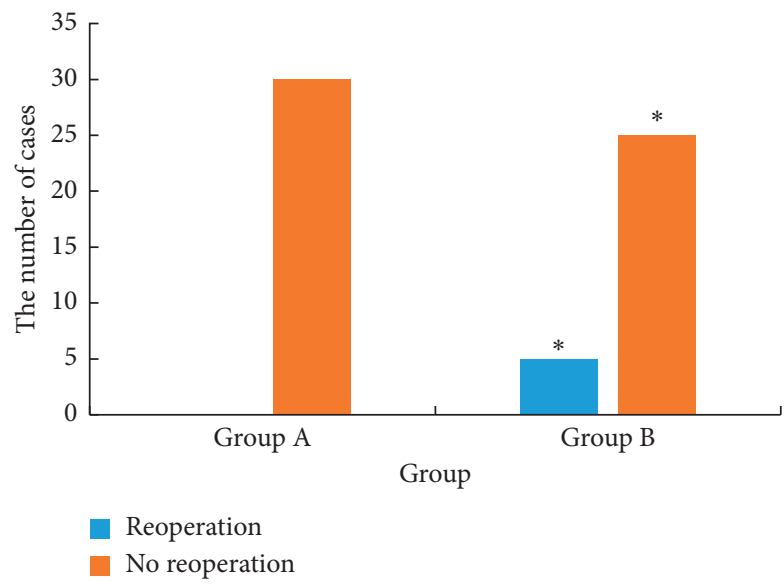

(a)

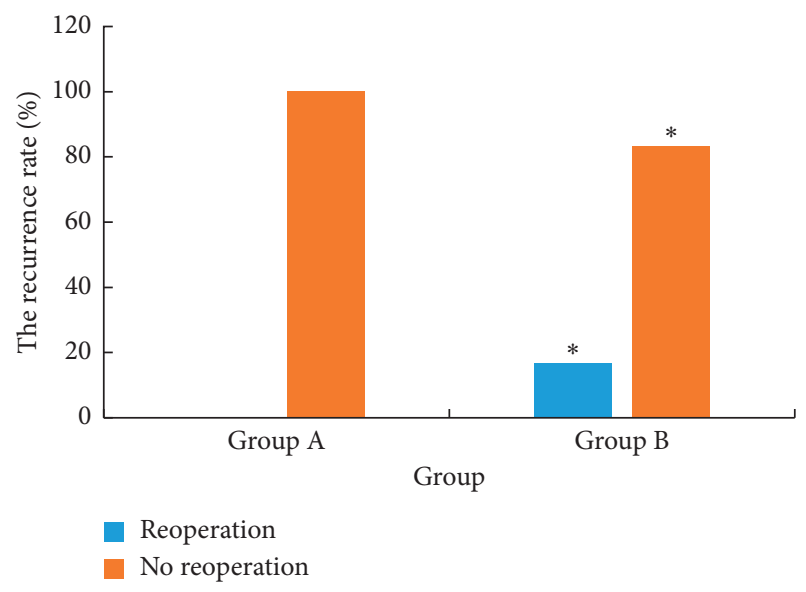

(b)

FiguRE 6: Comparison of the reoperation rate between the two groups. (a) Number of people. (b) Ratio. * means statistical significance compared with the experimental group, $P<0.05$.

It can be concluded that the BEMD algorithm had a high clinical application value in brain CT image processing.

Craniotomy hematoma removal is a clinically widely used $\mathrm{CDSH}$ surgical treatment. It has the advantages of clearing hematoma under direct vision and being able to deal with sudden situations during surgery. Clinical data showed that it has achieved good results in the treatment of CDSH. Anesthesia is a vital and indispensable part of CDSH hematoma removal. Anesthesia directly affects the prognosis of the patient and even the life safety of the patient. Propofol is currently an anesthetic widely used in clinical practice, which is often used as an anesthesia inducer during surgical 
treatment of CSDH patients. However, there is no definite conclusion on the effect of propofol on the brain. Studies pointed out that propofol can cause forgetting and even impair memory function. However, there are also opinions that propofol has no effect on the learning ability and memory function of experimental animals [20,21]. In this research, the $\mathrm{CDSH}$ patients were rolled into control group and experimental group. Both groups underwent hematoma removal. The experimental group was anesthetized with propofol, and the control group was anesthetized with conventional anesthesia. After the operation, the reoperation rate, hematoma volume, recurrence rate, and Markwalder grading results of the two groups were compared and analyzed. It was found that the recurrence rate and reoperation rate of the experimental group were lower compared with the control group, and the recovery of brain function and the disappearance of hematoma were faster. This indicated that the removal of craniotomy hematoma under propofol anesthesia had a high clinical value in the treatment of $\mathrm{CSDH}$.

\section{Conclusion}

In this research, the protective effect of hematoma removal under propofol anesthesia on the brain function of CSDH patients was analyzed based on the artificial intelligence algorithm analysis of the changes in CT image characteristics of $\mathrm{CSDH}$ patients. It was found that compared with patients in the craniotomy hematoma removal group under conventional anesthesia, patients in the hematoma removal surgery group under propofol anesthesia had a faster postoperative hematoma disappearance, a lower recurrence rate, and a better recovery of neurological function. It was verified that craniotomy hematoma removal under propofol anesthesia had a relatively better therapeutic effect on $\mathrm{CSDH}$. This article provides a reference and basis for the application of craniotomy hematoma removal under propofol anesthesia and artificial intelligence algorithms in the treatment of CSDH. Due to the limited length and sample number, this research is not comprehensive and in-depth enough. A future study and work will expand the sample size to further study this issue in a comprehensive and in-depth way.

\section{Data Availability}

The data used to support the findings of this study are available from the corresponding author upon request.

\section{Conflicts of Interest}

The authors declare that there are no conflicts of interest.

\section{References}

[1] G. H. Huang, X. C. Li, L. Ren et al., "Take it seriously or not: postoperative pneumocephalus in CSDH patients?," British Journal of Neurosurgery, vol. 34, no. 3, pp. 284-289, 2020.

[2] J. Huang, C. Gao, J. Dong, J. Zhang, and R. Jiang, "Drug treatment of chronic subdural hematoma," Expert Opinion on Pharmacotherapy, vol. 21, no. 4, pp. 435-444, 2020.
[3] P. J. Hutchinson, E. Edlmann, D. Bulters et al., "British neurosurgical trainee research collaborative; dex-CSDH trial collaborators. Trial of dexamethasone for chronic subdural hematoma," New England Journal of Medicine, vol. 383, no. 27, pp. 2616-2627, 2020.

[4] D. C. Holl, V. Volovici, C. M. F. Dirven et al., "Dutch chronic subdural hematoma research group (DSHR). Pathophysiology and nonsurgical treatment of chronic subdural hematoma: from past to present to future," World Neurosurg, vol. 116, pp. 402-411, 2018.

[5] D. Fiorella and A. S. Arthur, "Middle meningeal artery embolization for the management of chronic subdural hematoma," Journal of Neurointerventional Surgery, vol. 11, no. 9, pp. 912-915, 2019.

[6] E. Edlmann, E. P. Thelin, K. Caldwell et al., "Dex-CSDH trial collaborative and BNTRC collaborative. Dex-CSDH randomised, placebo-controlled trial of dexamethasone for chronic subdural haematoma: report of the internal pilot phase," Scientific Reports, vol. 9, no. 1, 2019.

[7] J. Feghali, W. Yang, and J. Huang, "Updates in chronic subdural hematoma: epidemiology, etiology, pathogenesis, treatment, and outcome," World Neurosurg, vol. 141, pp. 339-345, 2020.

[8] J. S. Catapano, C. L. Nguyen, A. A. Wakim, F. C. Albuquerque, and A. F. Ducruet, "Middle meningeal artery embolization for chronic subdural hematoma," Frontiers in Neurology, vol. 11, Article ID 557233, 2020.

[9] A. Allison, E. Edlmann, A. G. Kolias et al., "Statistical analysis plan for the Dex-CSDH trial: a randomised, double-blind, placebo-controlled trial of a 2-week course of dexamethasone for adult patients with a symptomatic chronic subdural haematoma," Trials, vol. 20, no. 1, 2019.

[10] R. Jiang, S. Zhao, R. Wang et al., "Safety and efficacy of atorvastatin for chronic subdural hematoma in Chinese patients: a randomized ClinicalTrial," JAMA Neurol, vol. 75, no. 11, pp. 1338-1346, 2018.

[11] L. Greuter, K. Lutz, J. Fandino, L. Mariani, R. Guzman, and J. Soleman, "Drain type after burr-hole drainage of chronic subdural hematoma in geriatric patients: a subanalysis of the cSDH-Drain randomized controlled trial," Neurosurgical Focus, vol. 49, no. 4, 2020.

[12] Z. Lv, X. Li, and W. Li, "Virtual reality geographical interactive scene semantics research for immersive geography learning," Neurocomputing, vol. 254, no. sep.6, pp. 71-78, 2017.

[13] Z. H. Lv, X. M. Li, W. X. Wang, B. Y. Zhang, J. X. Hu, and S. Z. Feng, "Government affairs service platform for smart city," Future Generation Computer Systems, vol. 81, pp. 443451, 2018.

[14] S. P. Ban, G. Hwang, H. S. Byoun et al., "Middle meningeal artery embolization for chronic subdural hematoma," Radiology, vol. 286, no. 3, pp. 992-999, 2018.

[15] A. G. Kolias, E. Edlmann, E. P. Thelin et al., "British Neurosurgical Trainee Research Collaborative (BNTRC) and DexCSDH Trial Collaborators. Dexamethasone for adult patients with a symptomatic chronic subdural haematoma (DexCSDH) trial: study protocol for a randomised controlled trial," Trials, vol. 19, no. 1, 2018.

[16] K. Lutz, M. Kamenova, S. Schaedelin et al., "Time to and possible risk factors for recurrence after burr-hole drainage of chronic subdural hematoma: a subanalysis of the cSDH-drain randomized controlled trial," World Neurosurg, vol. 132, pp. e283-e289, 2019.

[17] C. I. Morin, C. Touchette, M. Lévesque, K. Effendi, D. Fortin, and D. Mathieu, "Chronic subdural hematoma: toward a new 
management paradigm for an increasingly complex population," Journal of Neurotrauma, vol. 35, no. 16, pp. 1882-1885, 2018.

[18] A. Agrawal, A. P. Hernandez, and L. R. S. Moscote, "Letter: subperiosteal vs subdural drain after burr-hole drainage of chronic subdural hematoma: a randomized clinical trial (cSDH-Drain-Trial)," Neurosurgery, vol. 85, no. 4, 2019.

[19] S. Ridwan, A. M. Bohrer, A. Grote, and M. Simon, "Surgical treatment of chronic subdural hematoma: predicting recurrence and cure," World Neurosurg, vol. 128, pp. e1010-e1023, 2019.

[20] M. Kamenova, K. Lutz, S. Schaedelin, J. Fandino, L. Mariani, and J. Soleman, "Subperiosteal versus subdural drain after burr-hole drainage under blood thinners: a subanalysis of the cSDH-drain rct," World Neurosurg, vol. 139, pp. e113-e120, 2020.

[21] J. Soleman, K. Lutz, S. Schaedelin et al., "In Reply: subperiosteal vs subdural drain after burr-hole drainage of chronic subdural hematoma: a randomized clinical trial (cSDH-Drain-Trial)," Neurosurgery, vol. 85, no. 4, pp. E797-E798, 2019. 